\title{
Case Report \\ Posterior Reversible Encephalopathy Syndrome due to High Dose Corticosteroids for an MS Relapse
}

\author{
Sarah A. Morrow, ${ }^{1}$ Robina Rana, ${ }^{2}$ Donald Lee, ${ }^{1}$ Terri Paul, ${ }^{2}$ and Jeffrey L. Mahon ${ }^{2}$ \\ ${ }^{1}$ Department of Clinical Neurological Sciences, University of Western Ontario, 339 Windermere Road, London, ON, \\ Canada N6A 5 A5 \\ ${ }^{2}$ Department of Medicine, University of Western Ontario, 339 Windermere Road, London, ON, Canada N6A 5A5
}

Correspondence should be addressed to Sarah A. Morrow; sarah.morrow@lhsc.on.ca

Received 7 March 2015; Revised 28 April 2015; Accepted 17 May 2015

Academic Editor: Mathias Toft

Copyright (C) 2015 Sarah A. Morrow et al. This is an open access article distributed under the Creative Commons Attribution License, which permits unrestricted use, distribution, and reproduction in any medium, provided the original work is properly cited.

\begin{abstract}
Increased blood pressure is a known adverse effect associated with corticosteroids but little is published regarding the risk with the high doses used in multiple sclerosis (MS). A 53-year-old female with known relapsing remitting MS presented with a new brainstem relapse. Standard of care treatment for an acute MS relapse, $1250 \mathrm{mg}$ of oral prednisone for 5 days, was initiated. She developed an occipital headache and dizziness and felt generally unwell. These symptoms persisted after treatment was complete. On presentation to medical attention, her blood pressure was $199 / 110 \mathrm{mmHg}$, although she had no history of hypertension. MRI changes were consistent with posterior reversible encephalopathy syndrome (PRES), demonstrating abnormal T2 signal in both thalami, the posterior occipital and posterior parietal white matter with mild sulcal effacement. As her pressure normalized with medication, her symptoms resolved and the MRI changes improved. No secondary cause of hypertension was found. This is the first reported case of PRES secondary to high dose corticosteroid use for an MS relapse without a history of hypertension and with no other secondary cause of hypertension identified. This rare complication should be considered in MS patients presenting with a headache or other neurological symptoms during treatment for a relapse.
\end{abstract}

\section{Introduction}

Posterior reversible encephalopathy syndrome (PRES) is a sporadic disease most commonly associated with an acute increase in blood pressure [1]. Presenting symptoms include headache, visual changes, vomiting, seizure, and confusion [2]. There are many medical conditions and medications that are known to increase the risk of acute hypertension, including corticosteroids (CR). We report a case of PRES due to high dose CR in an MS patient with an acute demyelinating event.

\section{Case}

A 53-year-old right handed female was diagnosed with relapsing remitting MS in 2011 after clinical relapses in 2006, 2010, and 2011 and an MRI consistent with demyelination, none of which were treated with CR. She was started on interferon $\beta$-1a $30 \mu \mathrm{g}$ IM weekly, a disease modifying therapy for MS, shortly after diagnosis. She had a sensory relapse in 2012 that resolved without CR treatment. Past medical history was significant for chronic calcific pancreatitis that was not autoimmune in nature; investigations by gastroenterology were negative and it was felt to be idiopathic in nature. There was no history of alcohol abuse or acute pancreatitis, despite a past history of a cholecystectomy. There was no known history of hypertension (HTN) and her blood pressure (BP) in the past was around $125 / 85 \mathrm{mmHg}$.

In June 2013 she presented with diplopia. A new intranuclear ophthalmoplegia (INO) was identified and she was diagnosed with a relapse. Treatment with high dose CR was offered and she was started on $1250 \mathrm{mg}$ oral prednisone for 5 days with no taper. On day one of CR, she noted insomnia, dizziness, general malaise, and a headache described as dull and gradual in onset. Upon completion of the pulse CR treatment, her symptoms persisted and she therefore came to the Emergency Department. She was not seen by a physician as she felt that the wait was too long and left. 
Over the following week, her headache worsened and she re-presented to the Emergency Department, describing the headache as starting in the occipital region and migrating over time to be holocephalic. The pain was rated as 10/10 but continued to be dull and aching. No nausea or vomiting, visual changes, or new neurologic symptoms were present. Her BP was $199 / 110 \mathrm{mmHg}$ and heart rate was 78 beats per minute and regular and general medical examination was within normal limits. The neurologic examination was consistent with the findings prior to the onset of the relapse; her INO and diplopia had resolved. Investigations in the Emergency Department included a normal electrocardiogram with normal sinus rhythm and no evidence of left ventricular hypertrophy, normal urinalysis including no protein or blood, and unremarkable pelvic/renal ultrasound including no renal calculi. An MRI of the head and Cspine was performed. In addition to T2 hyperintensities in the brain and the cervical cord consistent with MS, unchanged since the previous MRI six months earlier, there was also abnormal T2 signal in both thalami, the posterior occipital and posterior parietal white matter extending into the vertex. Mild local sulcal effacement associated with these new lesions was also noted. There was no diffusion restriction noted with these lesions. The findings described above were consistent with a diagnosis of PRES (Figure 1). She was admitted to hospital; her BP was monitored and remained relatively unchanged for the next 48 hours despite treatment with hydrochlorothiazide and amlodipine. Her BP subsequently responded to labetalol and amlodipine and was stabilized at approximately $130 / 85 \mathrm{mmHg}$ and her headache resolved. Investigations for other causes of PRES as well as secondary causes of HTN were performed. Laboratory investigations included serum sodium $135 \mathrm{mmol} / \mathrm{L}$, serum potassium $3.0 \mathrm{mmol} / \mathrm{L}$, serum creatinine $79 \mu \mathrm{mol} / \mathrm{L}$, serum urea $4.1 \mathrm{mmol} / \mathrm{L}$, serum calcium $2.21 \mathrm{mmol} / \mathrm{L}$, and serum albumin level $43 \mathrm{~g} / \mathrm{L}$. Her body mass index was $24 \mathrm{~kg} / \mathrm{m}^{2}$, she did not have signs of Cushing's syndrome or thyroid gland disease, there were no changes of hypertensive retinopathy, and kidney function was normal. Anti-nuclear antibody (ANA) was negative. A urinalysis was negative for protein and hematuria. She was not pregnant. Her medications at presentation included nortriptyline and interferon B-1a only, and she was on no over-the-counter medications that affect BP or are associated with PRES. A 24-hour urine collection for free cortisol and fractionated metanephrines level was done in hospital 19 days after her first dose of CR and showed increases in the cortisol $(942 \mathrm{nmol} / \mathrm{d})$ and normetanephrine ( $666 \mu \mathrm{mol} / \mathrm{mol}$ creatinine) levels but a normal metanephrine level $(78 \mu \mathrm{mol} / \mathrm{mol}$ creatinine). Three subsequent 24 -hour urine collections for fractionated metanephrines, fractionated catecholamines, and dopamine levels, including one collected 2 weeks after stopping nortriptyline, were normal, ruling out nortriptyline as a potential factor in her increased BP or PRES. A second 24-hour urine free cortisol 113 days after her first dose of CR was normal $(285 \mathrm{nmol} / \mathrm{d})$. Thus, no secondary causes of HTN or PRES were found.

Follow-up MRI one month later demonstrated resolution of the signal change in the posterior cerebral hemispheres,

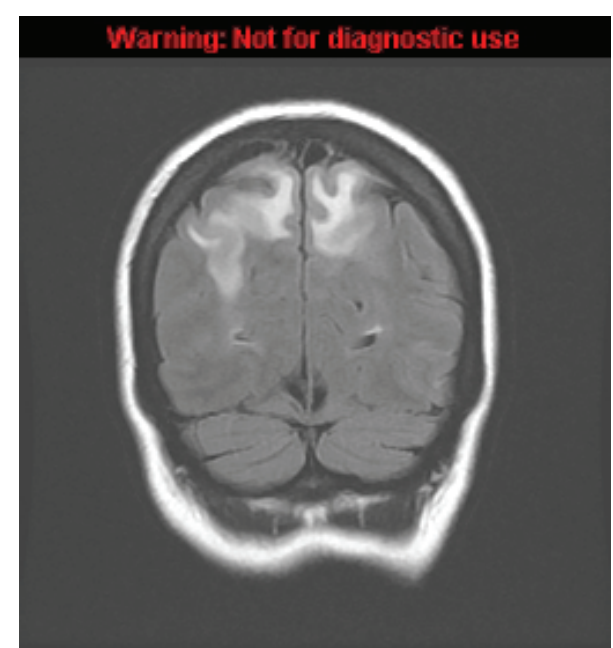

FIGURE 1: Coronal FLAIR image demonstrating hyperintensity in the posterior occipital white matter extending up to the vertex with mild local sulcal effacement consistent with posterior reversible encephalopathy syndrome (PRES).

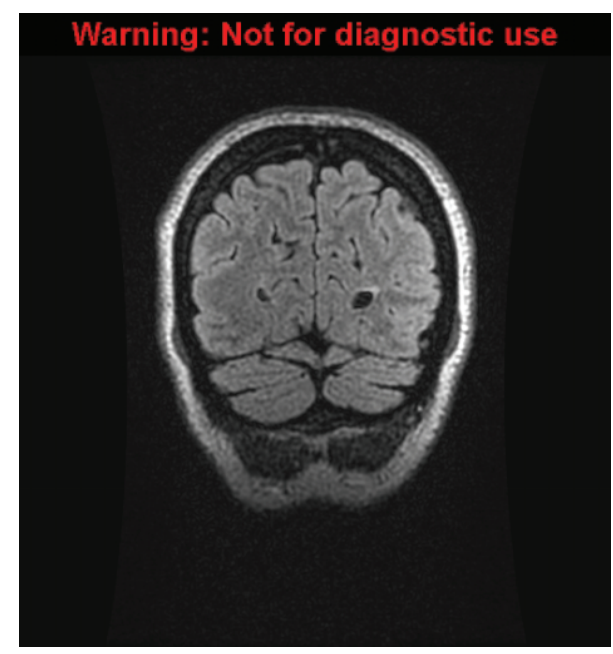

FIGURE 2: Follow-up coronal FLAIR image one month later demonstrating complete resolution of the hyperintensity noted previously.

consistent with resolution of PRES (Figure 2). She was discharged on amlodipine $10 \mathrm{mg}$ daily and labetalol $150 \mathrm{mg}$ twice a day. She continued to be neurologically stable at her followup visit in the MS clinic one month after discharge with a blood pressure of 140/87 $\mathrm{mmHg}$.

\section{Discussion}

PRES was first described by Hinchey et al. in 1996 but its true incidence is unknown [3]. Although the presenting symptoms can be severe, including visual loss, headaches, confusion, decreased level of consciousness, and/or seizures, and the syndrome is often completely reversible, there are some cases where full recovery has not occurred [2]. It is mainly associated with acute hypertension and with certain 
medical conditions such as electrolyte imbalance, vasoactive drugs, eclampsia/preeclampsia, sepsis, Guillain-Barre syndrome, autoimmune diseases and chemotherapy agents, and other immunosuppressive drugs [2, 4].

MRI is considered the gold standard for confirmation of diagnosis and typically demonstrates hyperintense T2 weighted and FLAIR lesions due to high water mobility, which appear normal on diffusion-weighted imaging predominantly occurring at the occipital regions [2]. Greater than $90 \%$ of patients demonstrate involvement of the parietooccipital lobes, but lesion can also be present in the frontal lobe basal ganglia, cerebellum, and brainstem in $1 / 3$ of cases [2]. PRES lesions tend to be symmetric, affecting both hemispheres. The PRES lesions are thought to represent vasogenic edema, although the pathophysiology that leads to PRES is not known; one popular theory is that acute hypertension is associated with failed autoregulation, enhanced vascular permeability, and hyperperfusion which leads to edema $[3,5]$. The mechanism underlying how hypertension can lead to PRES is not fully understood. It is postulated that acute hypertension is thought to exceed the upper limit of autoregulation, leading to vasodilation allowing leakage to occur leading to cerebral edema $[6,7]$.

The standard of care for acute multiple sclerosis (MS) relapses is high dose CR [8]. HTN is a known risk with the use of CR [9], possibly due to the effect of CR on the mineralocorticoid receptor [7]. There are several case reports of PRES being related to $\mathrm{CR}$ use. In most cases, however, the patient was also on another medication, such as CHOP or cisplatin chemotherapy for an underlying hematologic cancer $[10,11]$, or was acutely ill and normotensive [12] and thus a causal relationship between CR and PRES cannot be definitively made. There are two pediatric cases of PRES associated with pulses of high CR similar to our case, but in these cases, the patients were also acutely ill $[13,14]$. In a prospective study of MS patients treated with 1 gram of methylprednisolone daily for 3-5 days, only 4/64 developed HTN [15]. To our knowledge, this is the first case of PRES reported in an MS patient secondary to high dose CR treatment for an acute relapse without any other potential etiologies present. There have been cases of PRES associated with neuromyelitis optica spectrum disorders, but none in the context of CR use. The authors of that case series postulated that interference with the aquaporin channel by the aquaporin- 4 specific IgG may have contributed to the development of PRES [16].

It is well known that CR can cause HTN; a meta-analysis by Fardet et al. demonstrated an OR of 2.2 (95\% CI 1.4-3.8) for developing HTN in patients treated with CR compared to controls [17]. However, this meta-analysis included studies with low dose CR (5-40 mg) over a long period of time (months to years). The exception was one study by Chibane et al. where patients were treated with high dose pulse CR, 500-1000 mg methylprednisolone, similar to the dose used for MS relapses, for various eye diseases [18]. The authors reported HTN, defined as BP $\geq 180 / 110 \mathrm{mmHg}$, in only $3.4 \%$ of subjects. Whether these patients were symptomatic was not specified. None of the above studies noted cases of PRES associated with this increase in BP due to CR.
This rare complication should be considered in MS patients presenting with a headache or other neurological symptoms during CR treatment.

\section{Conflict of Interests}

The authors declare that there is no conflict of interests regarding the publication of this paper.

\section{References}

[1] V. H. Lee, E. F. M. Wijdicks, E. M. Manno, and A. A. Rabinstein, "Clinical spectrum of reversible posterior leukoencephalopathy syndrome," Archives of Neurology, vol. 65, no. 2, pp. 205-210, 2008.

[2] C. Roth and A. Ferbert, "The posterior reversible encephalopathy syndrome: what's certain, what's new?" Practical Neurology, vol. 11, no. 3, pp. 136-144, 2011.

[3] J. Hinchey, C. Chaves, B. Appignani et al., "A reversible posterior leukoencephalopathy syndrome," The New England Journal of Medicine, vol. 334, no. 8, pp. 494-500, 1996.

[4] C. J. Stevens and M. K. S. Heran, "The many faces of posterior reversible encephalopathy syndrome," British Journal of Radiology, vol. 85, no. 1020, pp. 1566-1575, 2012.

[5] G. Bo, L. Hui, L. Feng-li, and L. Cui, "Relationships between edema degree and clinical and biochemical parameters in posterior reversible encephalopathy syndrome: a preliminary study," Acta Neurologica Belgica, vol. 112, no. 3, pp. 281-285, 2012.

[6] Y. Li, D. Gor, D. Walicki et al., "Spectrum and potential pathogenesis of reversible posterior leukoencephalopathy syndrome," Journal of Stroke and Cerebrovascular Diseases, vol. 21, no. 8, pp. 873-882, 2012.

[7] W. Irvin, G. MacDonald, J. K. Smith, and W. Y. Kim, "Dexamethasone-induced posterior reversible encephalopathy syndrome," Journal of Clinical Oncology, vol. 25, no. 17, pp. 2484-2486, 2007.

[8] J. M. Burton, P. W. O'Connor, M. Hohol, and J. Beyene, "Oral versus intravenous steroids for treatment of relapses in multiple sclerosis," Cochrane Database of Systematic Reviews, no. 3, Article ID CD006921, 2009.

[9] E. Sarnes, L. Crofford, M. Watson, G. Dennis, H. Kan, and D. Bass, "Incidence and US costs of corticosteroid-associated adverse events: a systematic literature review," Clinical Therapeutics, vol. 33, no. 10, pp. 1413-1432, 2011.

[10] S. Tsukamoto, M. Takeuchi, C. Kawajiri et al., "Posterior reversible encephalopathy syndrome in an adult patient with acute lymphoblastic leukemia after remission induction chemotherapy," International Journal of Hematology, vol. 95, no. 2, pp. 204208, 2012.

[11] M. Hosoi, G. Yamamoto, Y. Imai, and M. Kurokawa, "Reversible posterior leukoencephalopathy syndrome following R-CHOP therapy for diffuse large B-cell lymphoma," Annals of Hematology, vol. 89, no. 2, pp. 207-208, 2010.

[12] M. T. Nguyen, I. Y. Virk, L. Chew, and J. L. Villano, "Extended use dexamethasone-associated posterior reversible encephalopathy syndrome with cisplatin-based chemotherapy," Journal of Clinical Neuroscience, vol. 16, no. 12, pp. 1688-1690, 2009.

[13] S. Kumar, A. Abhayambika, A. N. E. Sundaram, and J. A. Sharpe, "Posterior reversible encephalopathy syndrome presenting as balint syndrome," Journal of Neuro-Ophthalmology, vol. 31, no. 3, pp. 224-227, 2011. 
[14] F. Incecik, M. Ö. Hergüner, D. Yildizdaş et al., "Posterior reversible encephalopathy syndrome due to pulse methylprednisolone therapy in a child," Turkish Journal of Pediatrics, vol. 55, no. 4, pp. 455-457, 2013.

[15] V. Shaygannejad, F. Ashtari, M. Alinaghian, R. Norouzi, M. Salari, and F. Fatehi, "Short-term safety of pulse steroid therapy in multiple sclerosis relapses," Clinical Neuropharmacology, vol. 36, no. 1, pp. 1-3, 2013.

[16] S. M. Magaña, M. Matiello, S. J. Pittock et al., "Posterior reversible encephalopathy syndrome in neuromyelitis optica spectrum disorders," Neurology, vol. 72, no. 8, pp. 712-717, 2009.

[17] L. Fardet, A. Kassar, J. Cabane, and A. Flahault, "Corticosteroidinduced adverse events in adults: frequency, screening and prevention," Drug Safety, vol. 30, no. 10, pp. 861-881, 2007.

[18] S. Chibane, S. Feldman-Billard, I. Rossignol, R. Kassaei, F. Mihoubi-Mantout, and E. Héron, "Short-term tolerance of three days pulse methyprednisolone therapy: a prospective study in 146 patients," Revue de Medecine Interne, vol. 26, no. 1, pp. 20-26, 2005. 


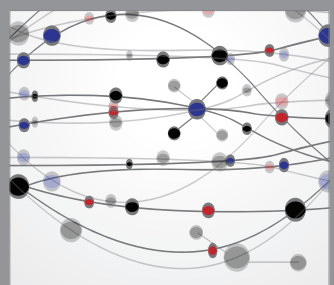

The Scientific World Journal
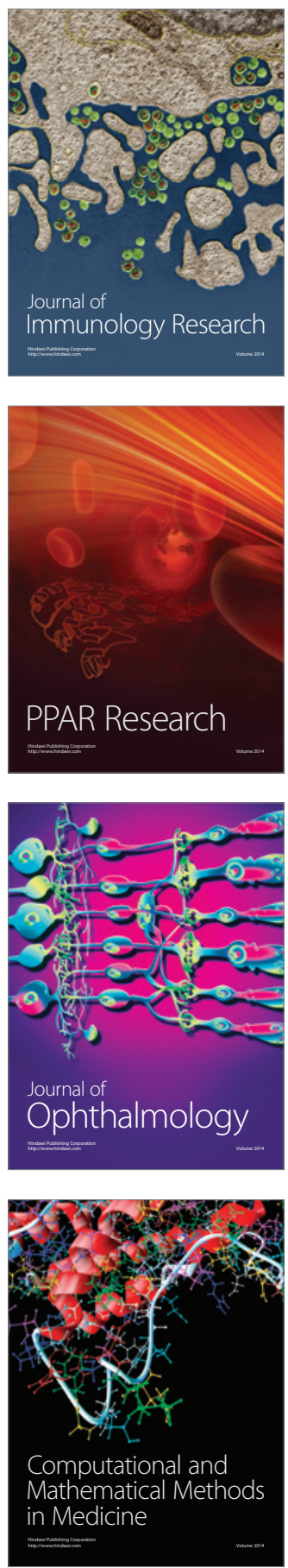

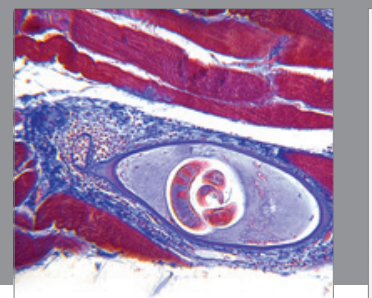

Gastroenterology

Research and Practice
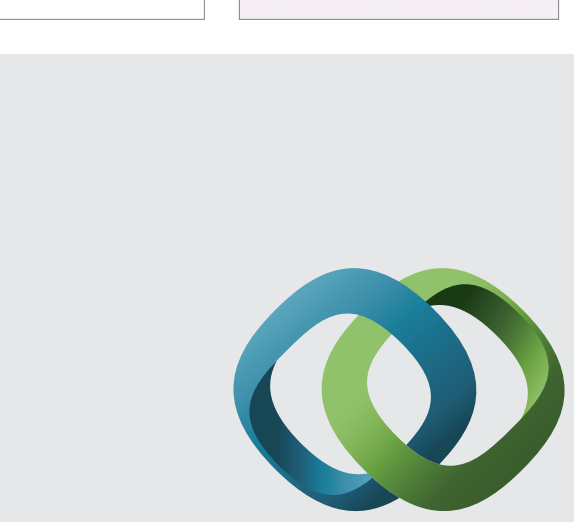

\section{Hindawi}

Submit your manuscripts at

http://www.hindawi.com
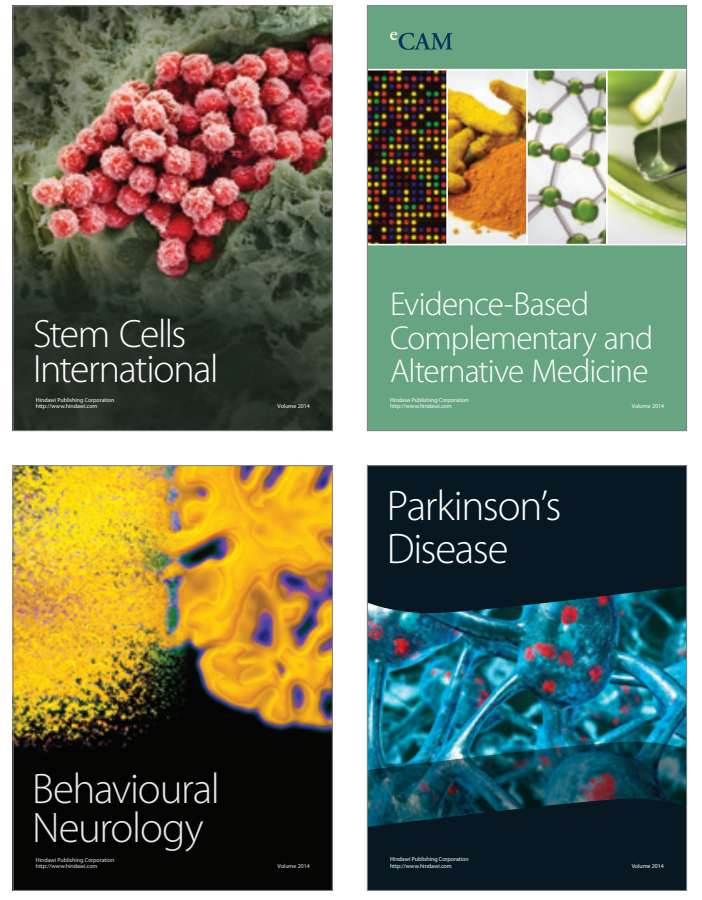
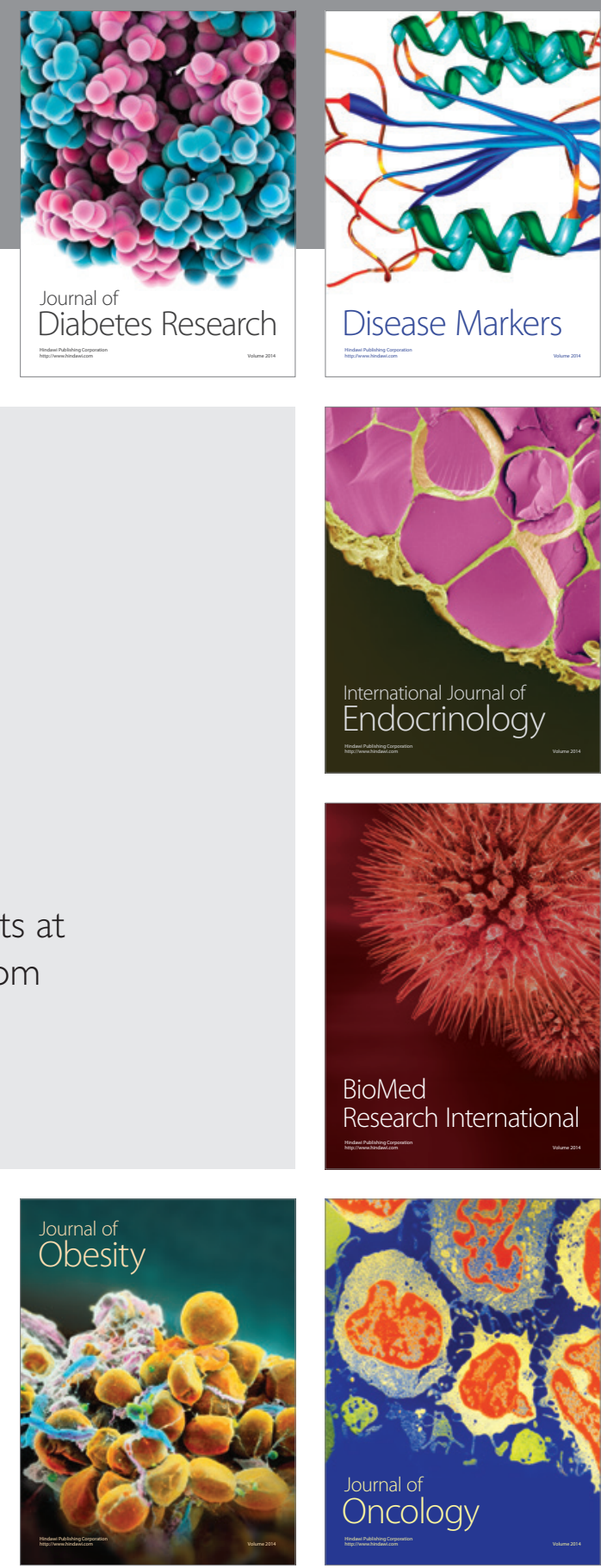

Disease Markers
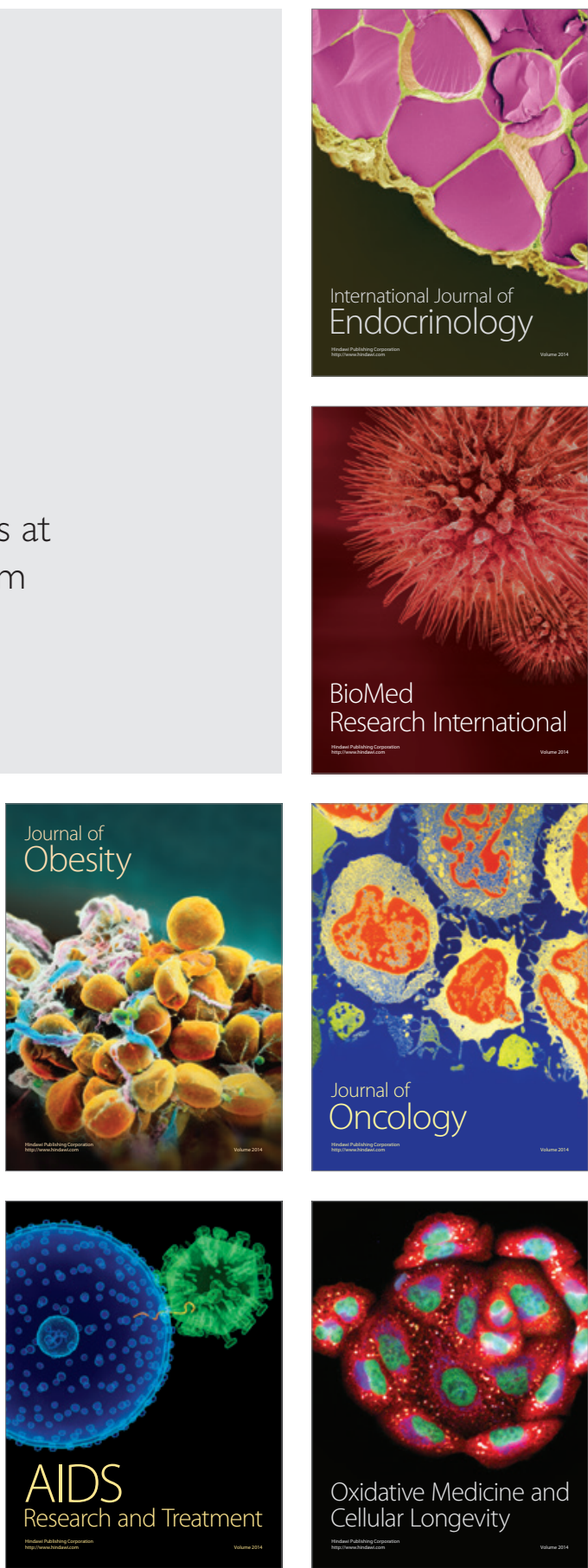